\title{
Pseudogap Phenomena in the BCS Pairing Model
}

\author{
Satoshi Fujimoto \\ Department of Physics, Kyoto University, Kyoto 606-8502, Japan
}

(November 8, 2018)

\begin{abstract}
We investigate pseudogap phenomena realized in the BCS pairing model with a long but finite interaction range. We calculate the single-particle self-energy in all orders exactly in the temperature range where the superconducting fluctuation propagator is Gaussian-like. It is found that vertex corrections to the self-energy, which are discarded in the previous studies, are crucially important for the pseudogap of the single-particle density of states in higher order calculations.
\end{abstract}

It is known that a pseudogap of the single-particle density of states (DOS) is a universal phenomenon observed in electron systems interacting with bosonic critical fluctuations. 8 \% The interaction with a Gaussian fluctuation which arises near the instability toward a long-range order like antiferromagnetism, charge density wave, or superconductivity results in a much enhanced damping of single-particle excitations, leading to a decrease of the DOS at the Fermi level. Recently, it has been proposed by some author that the pseudogap phenomena observed in high $T_{c}$ cuprates may be attributed to the superconducting fluctuation. 20 They have succeeded in mxtaining the pseudogap observed

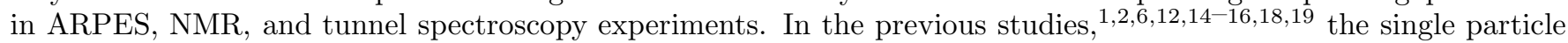
self-energy is calculated by one-loop approximation ( $t$-matrix approximation, diagrammatically expressed as Fig.1(a)) or self-consistent $t$-matrix approximation (expressed as Fig.1(b)). Although these calculations show qualitative agreement with experimental observations, there are some important differences between the results obtained by different approximation methods. For example, according to refs. 16 and 18, in the self-consistent one-loop approximation, the pseudogap of the DOS is destroyed near the Fermi level, and the quasiparticle peak is restored. On the other hand, such a restoration is not seen in the lowest-order one-loop approximation 19.196 Thus, it is desirable to examine the effects of vertex corrections, which are expressed as Fig.1(c), and previously discarded without any justification of the approximation. Moreover, the previous studies suggest that the substantial decrease of the DOS at the Fermi level appears for the interaction stronger than the intermediate coupling. For this strong interaction, the validity of the $t$-matrix approximation is obscure. Motivated by these considerations, here, we investigate a simple model for which we can calculate all-order self-energy corrections in the temperature range where the superconducting fluctuation is Gaussian-like.

(a)

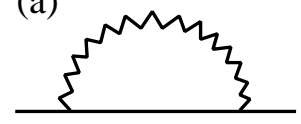

(c)

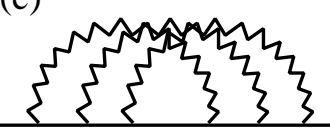

(b)

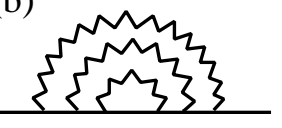

FIG. 1. Diagrams for the irreducible single-particle self-energy. The solid line represents the single electron Green's function. The wavy line represents the propagator of the superconducting fluctuation. (a) Lowest order one loop correction. (b) Self-consistent one loop approximation. (c) Some typical diagrams of vertex corrections which are not included in the diagrams (a) and (b).

The model Hamiltonian is given by

$$
\begin{aligned}
& H=\sum_{k, \sigma} E_{k} c_{k \sigma}^{\dagger} c_{k \sigma}-\frac{1}{N} \sum_{q} V(q) B^{\dagger}(q) B(q), \\
& V(q)=V \prod_{i=1}^{d}\left[\frac{1}{\pi} \frac{l_{c}}{1+l_{c}^{2} q_{i}^{2}}\right]
\end{aligned}
$$

where $B(q)=\sum_{k} \xi_{k} c_{k \downarrow} c_{-k+q \uparrow}$, and $d$ is the spatial dimension 21 The first term of eq.(11) is the kinetic energy with 
the energy dispersion $E_{k}$. The second term is the pairing attractive interaction with the interaction range $l_{c}$. $B(0)$ is a local annihilation operator of a Cooper pair with a structure factor $\xi_{k}$. For example, $\xi_{k}=1$ for $s$-wave pairing, $\xi_{k}=\sin k_{x}, \sin k_{y}$ for $p$-wave pairing, and $\xi_{k}=\cos k_{x}-\cos k_{y}, \cos k_{x} \cos k_{y}$ for $d$-wave pairing, and so forth. In the limit of $l_{c} \rightarrow+\infty$ the second term of eq.(1i) is reduced to $V B^{\dagger}(0) B(0)$. Then eq.(1) is the pairing model, which is exactly solvable.22 24 In this limit, the BCS mean field solution is exact, and the effects of fluctuation are completely suppressed. For finite but sufficiently large $l_{c}$, the superconducting fluctuation is restored, and can be calculated systematically using the expansion in terms of $1 / l_{c}^{d}$. We should bear in mind that although in this paper $l_{c}^{d}$ is taken as a large parameter, $V l_{c}^{d} \equiv U$ must be a finite constant even in the limit of $l_{c} \rightarrow+\infty$ in order to keep the mean field transition temperature finite, and $U$ is not a quantity of order $O\left(l_{c}^{d}\right)$.

Superconducting fluctuation propagator - We first discuss the propagator of the fluctuating bosonic field. For this aim, following Krishnamurthy and Shastry 24 we introduce the auxiliary boson field $\phi(q, \tau)$, and rewrite the interaction term of the action as

$$
\begin{aligned}
\sum_{q} \int_{0}^{\beta} d \tau \frac{V(q)}{N} B^{\dagger}(q, \tau) B(q, \tau) & \rightarrow \sum_{q} \int_{0}^{\beta} d \tau \phi^{*}(q, \tau) \phi(q, \tau) \\
& -\mathrm{i} \sum_{q} \sqrt{\frac{V(q)}{N}} \int_{0}^{\beta} d \tau\left[\phi^{*}(q, \tau) B(q, \tau)+\text { h.c. }\right] .
\end{aligned}
$$

Integrating the fermion fields, we obtain the action for the superconducting order parameter fluctuation.

$$
\begin{aligned}
S & =\sum_{q, m}\left(t+b q^{2}+a\left|\omega_{m}\right|\right) \phi_{q, m}^{*} \phi_{q, m} \\
& +c \sum_{\substack{m_{1}, m_{2}, m_{3}, k, k^{\prime}, q}} \phi_{k^{\prime}+q, m_{2}+m_{3}}^{*} \phi_{k-q, m_{1}-m_{3}}^{*} \phi_{k, m_{1}} \phi_{k^{\prime}, m_{2}}+O\left(\left(1 / l_{c}^{d}\right)^{2}\right) .
\end{aligned}
$$

Here, $\phi_{q, m}$ is the Fourier transform of $\phi(q, \tau) . t=\left(T-T_{c}\right) / T_{c}, a=3 \zeta(2) N(0) U /\left(4 \pi T_{c}\right), b=7 \zeta(3) N(0) U v_{F}^{2} /\left(32 \pi^{2} T_{c}^{2}\right)$, and $c=7 \zeta(3) N(0) U /\left(16 \pi^{2} l_{c}^{d} T_{c}^{2}\right)$ with $T_{c}$ the mean field transition temperature, and $N(0)$ the unrenormalized DOS at the Fermi level. In the derivation of the quadratic term, we assumed that the renormalization of the chemical potential caused by the superconducting fluctuation is negligibly small. In a two dimensional (2D) case, at sufficiently low temperatures, the chemical potential may be strongly renormalized to a negative value, and eventually, the crossover to the Bose-Einstein condensation occurs at the zero temperature. 25 In this paper, we do not consider such a low-temperature region in the $2 \mathrm{D}$ case. We also ignore the momentum and frequency dependencies of the coefficient of the quartic term $c$. The momentum dependence is negligible, since it is of a higher order in $1 / l_{c}^{d}$. The energy dependence is also negligible in the low energy region $\omega \ll E_{F}$. Note that $c$ is of the order $\sim 1 / l_{c}^{d}$. Thus, in the limit of $l_{c} \rightarrow+\infty$, the Gaussian term (the first term of eq.(画) dominates, and the propagator of the boson field is given by

$$
\left\langle\phi_{q, n} \phi_{q, n}^{*}\right\rangle=\frac{1}{a\left|\omega_{n}\right|+b q^{2}+t} .
$$

This result was previously obtained by Krishnamurthy and Shastry 24 In this limit, the BCS mean field solution is exact, and as a result, the single-particle self-energy correction vanishes.

To compute the effects of superconducting fluctuation on the self-energy, we consider finite but very large $l_{c}$. Then, we need to take into account the effects of the quartic and higher order terms of eq.(任). In the following, we consider only the quartic term, since it gives leading corrections. Applying the standard renormalization group method,26 we obtain the one-loop scaling equations,

$$
\begin{aligned}
& \frac{d t}{d l}=2 t+\frac{a_{0} c}{1+t}, \\
& \frac{d c}{d l}=(2-d) c-\frac{b_{0} c^{2}}{(1+t)^{2}} .
\end{aligned}
$$

Here, $a_{0}$ and $b_{0}$ are positive constants. From eq.(7), we see that for $d \geq 2$, the quartic term is irrelevant or marginally irrelevant. The main effect of the quartic term is the renormalization of the transition temperature, which is estimated from eq.(6). The coefficients $a$ and $b$ of eq.(5) are renormalized by higher order corrections than two loop. Thus, if the Ginzburg criterion for the smallness of fluctuations is satisfied by the initial values of the parameters, $t, a, b$, and $c$, the Gaussian-type propagator eq.(5) with the renormalized $t, a$, and $b$ can be regarded as the exact propagator of 
the superconducting fluctuation. Now we consider the case of $t \gg b l_{c}^{-2}$ in which, as will be shown later, the all-order self-energy corrections can be calculated exactly. In this case, the Ginzburg criterion is given by

$$
\left(\frac{4 c T_{c}}{l_{c}^{d}}\right)^{\frac{1}{2}} \ll t
$$

Since $c \sim O\left(1 / l_{c}^{d}\right)$, the condition eq.(\$) is satisfied when $l_{c}$ is sufficiently large to satisfy $t \gg b l_{c}^{-2}$. In the following, we consider only the situation wherein the unrenormalized coefficients $a, b, c$, and $t$ satisfy the Ginzburg criterion eq.(8), and that eq.(5) with the renormalized parameters is a good approximation for the correlation function of the superconducting fluctuation.
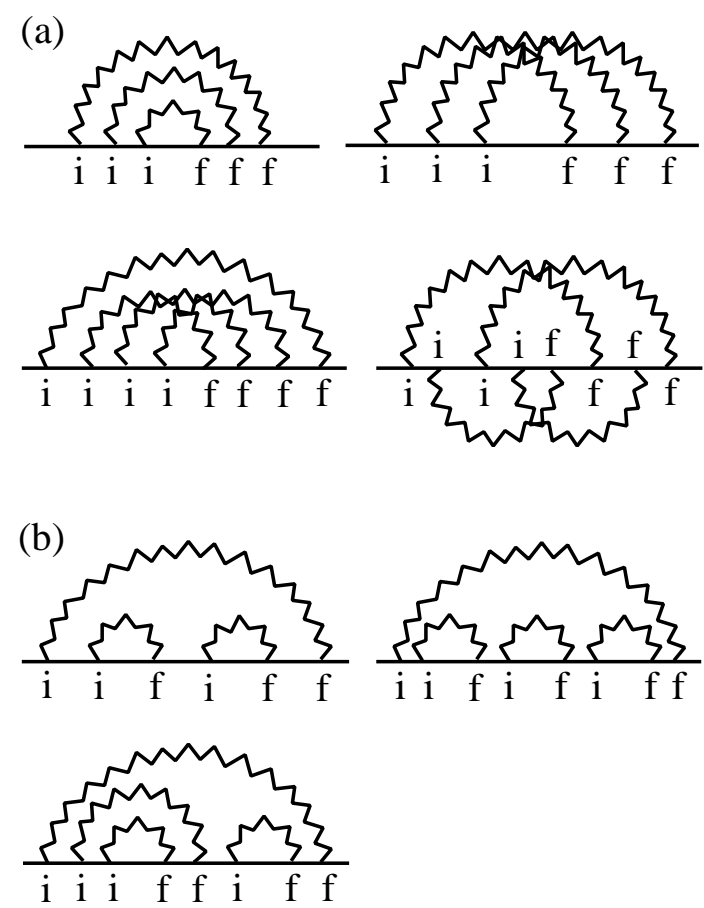

FIG. 2. (a) Some typical self-energy diagrams in which all initial vertices are on the left of all final vertices. The symbol $i$ represents the initial vertex, and $f$ the final vertex. (b) Some examples of diagrams which are not included in $\Sigma_{k}^{(n)^{\prime}}(\varepsilon)$.

All-order self-energy corrections - We expand the irreducible single-particle self-energy in terms of $1 / l_{c}^{d}$,

$$
\Sigma_{k}(\varepsilon)=\sum_{n=1}^{\infty} \Sigma_{k}^{(n)}(\varepsilon)
$$

Here, $\Sigma_{k}^{(n)}(\varepsilon)$ is the $n$-th order term in $1 / l_{c}^{d}$. $\Sigma_{k}^{(n)}(\varepsilon)$ consists of two parts: one part is the contribution from the diagrams in which all initial vertices are on the left of all final vertices as shown in Fig.2(a), and the other part is the contribution from other irreducible diagrams as shown in Fig.2(b). We express the former one as $\Sigma_{k}^{(n)^{\prime}}(\varepsilon)$. Carrying out the frequency summation analytically, we obtain

$$
\begin{aligned}
\Sigma_{k}^{(n)^{\prime}}(\varepsilon)= & \sum_{\{P\}} \frac{1}{N^{n}} \sum_{q_{1}, \ldots, q_{n}} \prod_{m=1}^{n} \frac{g_{k}\left(q_{m}\right)}{\mathrm{i} \varepsilon+(-1)^{m-1} E_{k-\sum_{j=1}^{m} q_{j}}+\mathrm{i} \sum_{j=1}^{m} C\left(q_{j}\right) \operatorname{sgn} \varepsilon} \\
& \times \prod_{m^{\prime}=1}^{n} \frac{1}{\mathrm{i} \varepsilon+(-1)^{m^{\prime}-1} E_{k-\sum_{j=1}^{m^{\prime}} q_{P_{j}}}+\mathrm{i} \sum_{j=1}^{m^{\prime}} C\left(q_{P_{j}}\right) \operatorname{sgn} \varepsilon},
\end{aligned}
$$

where $g_{k}(q)=T \xi_{k}^{2} V(q) /\left(t+b q^{2}\right)$, and $C(q)=\left(t+b q^{2}\right) / a .\{P\} \equiv\left\{P_{1}, P_{2}, \ldots, P_{n}\right\}$ is generated by a permutation of $\{1,2, \ldots, n\}$ under the rule that permutations between numbers with different parities are not allowed. For example, $\{P\}=\{1,2,3\},\{3,2,1\}$ for $n=3$, and $\{P\}=\{1,2,3,4\},\{3,2,1,4\},\{1,4,3,2\},\{3,4,1,2\}$ for $n=4$, and so forth. $\sum_{\{P\}}$ is the summation over these permutations. 
In general, we cannot carry out $q$-summation of eq.(10) analytically. However, for $t \gg b / l_{c}^{2}$, eq.(10) can be greatly simplified. As we will see later, this case is very important for us. In this limit, we can replace $q$-summation of eq.(10), $\frac{1}{N} \sum_{q}$, with $\left(1 / l_{c}^{d}\right) \sum_{q} \delta_{q, 0}$, since the momentum transferred is restricted within $q<1 / l_{c} \ll k_{F}$, for which $g_{k}(q) \approx g_{k}(0), C(q) \approx C(0)$, and $E_{k-q} \approx E_{k}$. In this approximation, all crossing diagrams of $\Sigma_{k}^{(n)^{\prime}}(\varepsilon)$ give the same contribution as its non-crossing diagrams. This means that vertex corrections which are not included in self-consistent $t$-matrix approximation are not negligible. The total number of these diagrams, $N(n)$, is $m !(m+1)$ ! for $n=2 m+1$, and $(m !)^{2}$ for $n=2 m$. Thus, we can rewrite eq. (10) as,

$$
\Sigma_{k}^{(n)^{\prime}}(\varepsilon)=N(n) \tilde{g}_{k}^{n} \frac{1}{\mathrm{i} \varepsilon+(-1)^{n-1} E_{k}+\mathrm{i} n C(0) \operatorname{sgn} \varepsilon} \prod_{m=1}^{n-1} \frac{1}{\left(\mathrm{i} \varepsilon+(-1)^{m-1} E_{k}+\mathrm{i} m C(0) \operatorname{sgn} \varepsilon\right)^{2}},
$$

where $\tilde{g}_{k}=g_{k}(0) / l_{c}^{d}$. This expression has the same form as that obtained by Sadovskii for one-dimensional fermion systems interacting with static Gaussian fluctuation. 6 Then, we can apply Elyutin-Sadovskii's combinatorics method to evaluate all diagrams. According to Sadovskii, the contributions from $\Sigma_{k}^{(n)}(\varepsilon)-\Sigma_{k}^{(n)^{\prime}}(\varepsilon)$ are expressed in terms of $\Sigma_{k}^{(m)^{\prime}}(\varepsilon)$ with $m<n$. $⿴$ Using Elyutin-Sadovskii's method, we can write the self-energy in all orders as a continued fraction form,

$$
\Sigma_{k}(\varepsilon)=\frac{v(1) \tilde{g}_{k}}{S_{1}(k, \varepsilon)-\frac{v(2) \tilde{g}_{k}}{S_{2}(k, \varepsilon)-\frac{v(3) \tilde{g}_{k}}{S_{3}(k, \varepsilon)-\frac{v(4) \tilde{g}_{k}}{S_{4}(k, \varepsilon)-} \cdot}}},
$$

where $S_{m}(k, \varepsilon)=\mathrm{i} \varepsilon+(-1)^{m-1} E_{k}+\mathrm{i} m C(0) \operatorname{sgn} \varepsilon$, and $v(m)=[(m+1) / 2],[\ldots]$ is Gauss's symbol. From this analytical expression, we obtain the single-particle DOS, $\rho(\varepsilon)=-1 / \pi \sum_{k} \operatorname{Im}\left[1 /\left(\varepsilon-E_{k}-\Sigma_{k}^{R}(\varepsilon)\right)\right]$. We calculate $\rho(\varepsilon)$ numerically in the case of $s$-wave pairing $\xi_{k}=1$, replacing $\sum_{k}$ with $N(0) \int d \varepsilon_{k}$. The result is shown in Fig.3(a). The parameters are chosen so as to satisfy the condition eq.(8) and $b l_{c}^{-2} \ll t$. We see that the pseudogap appears near the Fermi level. We also show the energy dependence of the self-energy in Figs.3(b) and 3(c). It is seen that the scatterig rate, the imaginary part of the self-energy, is much enhanced near the Fermi leyel, and that the real part has a positive slope for $\varepsilon \sim 0$. These features are characteristic of the pseudogap state.6.1.18 A similar result is obtained for other pairing symmetries, such as $p$-wave, $d$-wave, and so forth. 

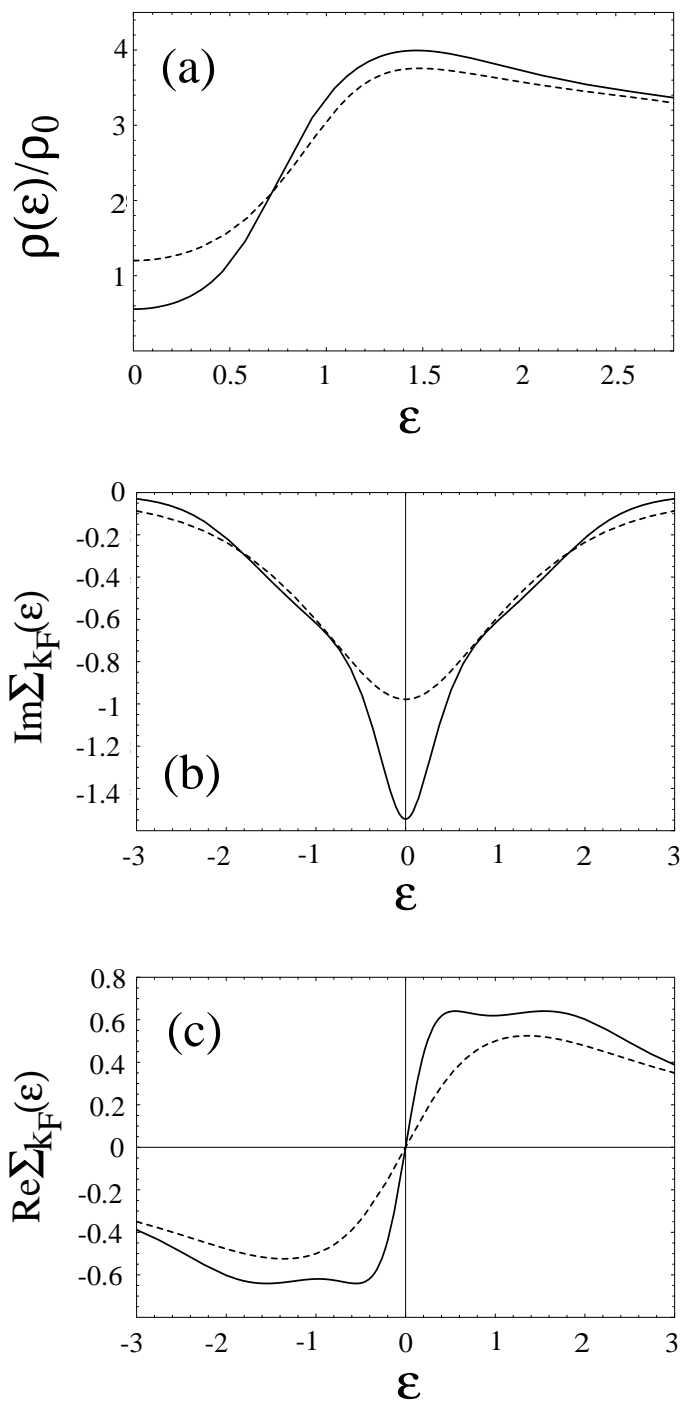

FIG. 3. (a) The single particle DOS $\rho(\varepsilon)$ plotted as a function of energy. The vertical axis is renormalized by the bare DOS $\rho_{0} . \quad \tilde{g}=1, C(0)=0.1$ for the solid line. $\tilde{g}=1, C(0)=0.5$ for the dotted line. (b) The imaginary part of the self-energy at the Fermi momentum plotted as a function of energy. The same parameters as Fig.3(a) are used. (c) The real part of the self-energy at the Fermi momentum plotted as a function of energy. The same parameters as above are used.

Analytical results for single-particle damping - The pseudogap behavior is due to the large enhancement of the damping, which is caused by strong scattering with superconducting fluctuation. Here, we will see how this enhancement is affected by the vertex corrections to the self-energy which were discarded in the previous studies. For this purpose, we calculate analytically the damping of electrons at the Fermi level,

$$
\gamma \equiv-\operatorname{Im} \Sigma_{k_{F}}^{R}(0)=\frac{v(1) \tilde{g}_{k}}{C(0)+\frac{v(2) \tilde{g}_{k}}{2 C(0)+\frac{v(3) \tilde{g}_{k}}{3 C(0)+\frac{v(4) \tilde{g}_{k}}{4 C(0)+\ddots}}}}
$$

The limiting value of this continued fraction is obtained from the recursion relation, $P_{n+1}=(n+1) C(0) P_{n}+v(n+7$ 2) $\tilde{g} P_{n-1}, Q_{n+1}=(n+1) C(0) Q_{n}+v(n+2) \tilde{g} Q_{n-1}, P_{0}=0, Q_{0}=1, P_{-1}=1, Q_{-1}=0$, and $\gamma=\lim _{n \rightarrow \infty} P_{n} / Q_{n} .27$ Up to a numerical constant, we have $\gamma \sim \tilde{g}_{k} / C(0)=a T U /\left(\pi^{d} l_{c}^{d} t^{2}\right)$. For $\sqrt{a U /\left(\pi^{d} l_{c}^{d}\right)} \gg t$, the damping of electrons is much enhanced, $\sim T / t^{2} \gg T$. Since we consider the case of $t \gg b / l_{c}^{2}$, for which the Ginzburg criterion eq.(8) is also satisfied, the temperature range where the above result is applicable is $b / l_{c}^{2} \ll t \ll \sqrt{a U /\left(\pi^{d} l_{c}^{d}\right)}$. It is noted that 
the result of all-order calculation gives the same asymptotic behavior as that obtained by the lowest-order one-loop approximation which is also given by $\sim \tilde{g} / C(0) \sim T / t^{2}$. This implies that higher order corrections cancel each other, and the lowest-order term gives the leading contribution. This cancellation is not due to the presence of the Ward identity. We do not have the Ward identity which relates a self-energy to a vertex in particle-particle channel. Actually, the cancellation takes place among different order diagrams. The total number of the diagrams which give dominant contribution in the $n$-th order increases as $O\left((n !)^{2}\right)$. As a result, higher order contributions cancel with lower order contributions effectively. We compare this result with that obtained from self-consistent $t$-matrix approximation. The self-energy in the self-consistent approximation is derived by substituting $v(m)=1$ for all $m$ of eq. 12 . The damping at the Fermi level, $\gamma_{s c}$, is given by

$$
\gamma_{s c}=\frac{\tilde{g}_{k}}{C(0)+\frac{\tilde{g}_{k}}{2 C(0)+\frac{\tilde{g}_{k}}{3 C(0)+\frac{\tilde{g}_{k}}{4 C(0)+\ddots}}}}=\frac{\mathrm{i} \sqrt{\tilde{g}_{k}} J_{1}\left(\mathrm{i} \frac{2 \sqrt{\tilde{g}_{k}}}{C(0)}\right)}{J_{0}\left(\mathrm{i} \frac{2 \sqrt{\tilde{g}_{k}}}{C(0)}\right)}
$$

where $J_{n}(x)$ is the $n$-th Bessel function. For sufficiently small $t$ (large $\sqrt{\tilde{g}_{k}} / C(0)$ ), eq.(14) is well approximated by $\sqrt{\tilde{g}_{k}}=\left[\xi_{k}^{2} U T /\left(t l_{c}^{d} \pi^{d}\right)\right]^{1 / 2} \sim 1 / \sqrt{t}$. This is much suppressed compared with the result of all-order calculations. For $t \gg b / l_{c}^{2}, \sqrt{\tilde{g}_{k}} \ll\left[\xi_{k}^{2} U T /\left(\pi^{d} b l_{c}^{d-2}\right)\right]^{1 / 2}$. Thus, in the case of $d=2$ or 3 , the damping $\gamma_{s c}$ cannot be enhanced sufficiently under this condition. This result presents a clear contrast with the all-order result. The self-consistent $t$-matrix approximation, which neglects vertex corrections underestimates crucially the effects of strong scattering by critical fluctuations, and may cause a fatal error not only quantitatively but also qualitatively in a pseudogap state. These results instructively show that the lowest-order one-loop calculation is more reliable than the self-consistent one-loop treatment, and furthermore imply that the lowest order one-loop treatment of the self-energy corrections may be a fairly good approximation because of the cancellation between higher order corrections.

In summary, we have studied the pseudogap of the single particle DOS in the BCS pairing model with a long but finite interaction range using a well controlled approximation method. We have derived the all-order single-particle self-energy exactly in the temperature range where the superconducting fluctuation propagator is a Gaussian form. It has been revealed that the vertex corrections to the self-energy are essentially important in higher order calculations.

The author would like to thank K. Yamada and B. S. Shastry for useful conversations. This work was partly supported by a Grant-in-Aid from the Ministry of Education, Culture, Science, and Technology, Japan.

${ }^{1}$ E. Abraham, M Redi, and C. Woo: Phys. Rev. B1 (1970) 218.

${ }^{2}$ J. P. Hurault and K. Maki: Phys. Rev. B2 (1970) 2560.

${ }^{3}$ M. J. Rice and S. Strässler: Solid State Communication 13 (1973) 1389.

${ }^{4}$ M. V. Sadovskii: Sov. Phys. JETP 50 (1979) 989.

${ }^{5}$ A. Kampf and J. R. Schrieffer: Phys. Rev. B41 (1990) 6399.

${ }^{6}$ C. Di Castro, et al.: Phys. Rev. B49 (1990) 10211.

7 J. J. Deisz, D. W. Hess, and J. W. Serene: Phys. Rev. Lett. 76 (1996) 1312.

${ }^{8}$ R. H. McKenzie and D. Scarratt: Phys. Rev. B54 (1996) 12709.

${ }^{9}$ M. Randeria et al.: Phys. Rev. Lett. 69 (1992) 2001; M. Randeria and A. A. Varlamov: Phys. Rev. B50 (1994) 10401.

${ }^{10}$ V. Emery and S. Kivelson: Nature 374 (1995) 434.

${ }^{11}$ V. B. Geshkenbein, L. B. Ioffe, and A. I. Larkin: Phys. Rev. B55 (1997) 3173.

12 O. Tchernyshyov: Phys. Rev. B56 (1997) 3372.

${ }^{13}$ B. Janko, J. Maly, and K. Levin: Phys. Rev. B56 (1997) 11407.

14 J. R. Engelbrecht et al.: Phys. Rev. B57 (1998) 13406.

15 A. A. Varlamov et al.: Adv. Phys. 48 (1999) 655.

${ }^{16}$ S. Moukouri et al.: Phys. Rev. B61 (2000) 7887;

${ }^{17}$ B. Kyung: Phys. Rev. B63 (2000) 014502.

${ }^{18}$ Y. Yanase and K. Yamada: J. Phys. Soc. Jpn. 70 (2001) 1659.

${ }^{19}$ D. Rohe and W. Metzner: Phys. Rev. B63 (2001) 224509. 
${ }^{20}$ B. Kyung, S. Allen, and A.-M. S. Tremblay: Phys. Rev. B64 (2001) 075116. The importance of vertex corrections for the pseudogap phenomena is also pointed out in this paper.

${ }^{21}$ A simlar but different model was also studied in K. Yang and S. L. Sondhi, Phys. Rev. B62 (2000) 11778.

${ }^{22}$ R. W. Richardson: Phys. Lett. 3 (1963) 277; J. Math. Phys. 18 (1977) 1802.

${ }^{23}$ M. C. Camiaggio, A. M. F. Rivas, and M. Saraceno: Nucl. Phys. A624 (1997) 157.

${ }^{24}$ H. R. Krishnamurthy and B. S. Shastry: Phys. Rev. Lett. 84 (2000) 4918.

${ }^{25}$ S. Schmitt-Rink, C. M. Varma, and A. E. Ruckenstein: Phys. Rev. Lett. 63 (1989) 445.

${ }^{26}$ For example, J. Zinn-Justin: Quantum Field Theory and Critical Phenomena (Oxford, New York, 1993); R. Shankar: Rev. Mod. Phys. 66 (1994) 129.

${ }^{27}$ H. S. Wall: Analytic Theory of Continued Fractions (Chelsea, New York, 1967). 